course, another matter. That is, CPET is a test modality, the fundamental purpose of which is to gradationally stress the physiological systems that have the potential to contribute to exercise intolerance in a patient.

With regard to the comment concerning exercise modality, the Task Force report has clearly addressed the issue of possible differences in the ventilatory and metabolic responses for cycle ergometry and shuttle walking in COPD [4, 5]. Furthermore, based on the available literature, the use of walking tests has been recommended for assessing the degree of arterial oxygen desaturation in patients with ILD. However, as treadmill protocols have not yet been used extensively for prognostic evaluation or for the assessment of interventions, the Task Force is of the opinion that it is not possible to formulate evidence-based recommendations for their use in clinical practice at this time.

In response to the issue of which measurements are essential, the Task Force report has clearly indicated those parameters and indices which have demonstrated a particular utility in: 1) functional evaluation, such as peak oxygen uptake $\left(V^{\prime} \mathrm{O}_{2}\right.$,peak); 2) prognostic evaluation, such as $V^{\prime} \mathrm{O}_{2}$,peak and $V^{\prime} \mathrm{E} / V^{\prime} \mathrm{CO}_{2}$; and 3) the evaluation of interventions, such as endurance time and "iso-time" measurements (e.g. ventilation, inspiratory capacity) during high-intensity, constant work-rate protocols.

With regard to the final point, again the Task Force report clearly states that measurement of the ventilatory response in exercise is an essential part of the functional and prognostic evaluation of patients with chronic lung and heart diseases and, if necessary, should be obtained in specialist centres.

J.E. Cotes and J.W. Reed suggest the use of $V^{\prime} E$ at an oxygen uptake $\left(V^{\prime} \mathrm{O}_{2}\right)$ of $1 \mathrm{~L} \cdot \mathrm{min}^{-1}\left(V^{\prime} \mathrm{E}, \mathrm{st}\right)$ as an appropriate and informative index of what they term the "ventilatory burden" of patients with lung disease. We feel that this deserves comment. This is because it provides what amounts to a single, and arbitrary, value on the profile of the ventilatory equivalent for oxygen $\left(V^{\prime} \mathrm{E} / V^{\prime} \mathrm{O}_{2}\right)$, the pattern of which is neither linear nor monotonic during exercise. Some subjects with lung disease (and, of course, normal but sedentary, and especially elderly, subjects) could be below the threshold of metabolic acidosis at a $V^{\prime} \mathrm{O}_{2}$ of $1 \mathrm{~L} \cdot \mathrm{min}^{-1}$, whereas others could be above the threshold. The value of $V^{\prime} \mathrm{E}$,st could, therefore, disguise important functional differences of $V^{\prime} \mathrm{E}$ response. Using $V^{\prime} \mathrm{CO}_{2}$ as the frame of reference overcomes many, although not all, of these concerns. The $V^{\prime} \mathrm{E}-V^{\prime} \mathrm{CO}_{2}$ relationship has been consistently demonstrated to be highly linear up to the respiratory compensation point and consequently, using either its linear characteristics or the minimum value of $V^{\prime} \mathrm{E} / V^{\prime} \mathrm{CO}_{2}$ (a justifiable physiological index reflecting the onset of respiratory compensation for the metabolic acidosis) is, we contend, more consonant with its physiological determinants.

In conclusion, we would like to thank J.E. Cotes and J.W. Reed for opening up a range of issues for debate that relate to clinical exercise testing. We hope that our responses to those that fall within the scope of the Task Force report are found to be constructive.

\section{P. Palange* and S.A. Ward}

*University of Rome "La Sapienza', Dept of Clinical Medicine, Pulmonary Function Unit, Rome Italy, and "Institute of Membrane and Systems Biology, University of Leeds, Leeds, UK.

\section{STATEMENT OF INTEREST}

None declared.

\section{REFERENCES}

1 Palange $\mathrm{P}$, Ward SA, Carlsen $\mathrm{K}-\mathrm{H}$, et al. Recommendations on the use of exercise testing in clinical practice. Eur Respir J 2007; 29: 185-209.

2 ERS Task Force on Standardization of Clinical Exercise Testing. Clinical exercise testing with reference to lung diseases: indications, standardisation and interpretation strategies. Eur Respir J 1997; 10: 2662-2689.

3 American Thoracic Society. American College of Chest Physicians. ATS/ACCP Statement on cardiopulmonary exercise testing. Am J Respir Crit Care Med 2003; 167: 211-277.

4 Palange $\mathrm{P}$, Forte S, Onorati P, Manfredi F, Serra P, Carlone S. Ventilatory and metabolic adaptations to cycling and walking in COPD. J Appl Physiol 2000; 88: 1715-1720.

5 Man WD, Soliman MG, Gearing J, et al. Symptoms and quadriceps fatigue after walking and cycling in chronic obstructive pulmonary disease. Am J Respir Crit Care Med 2003; 168: 562-567.

\title{
Hot and cold biopsy: implications of study design on
} \section{outcomes}

\section{To the Editors:}

We read with interest the article in the European Respiratory Journal by TREMBLAY et al. [1], wherein the authors conclude that the use of hot biopsy forceps for endobronchial biopsy does not appear to have a negative impact on the pathological samples, and that there was a statistically significant (albeit clinically insignificant) reduction in bleeding score with hot biopsy forceps. However, many of the conclusions of the study have limitations because of the study design of alternate hot and cold biopsies.

The authors state that the quantification of bleeding was carried out and recorded by the bronchoscopists between each biopsy on a four-point scale. However, the interval between the two 
biopsies was not stated. Moreover, it is a common observation that the ooze from an endobronchial lesion continues after the biopsy has been taken and thus it becomes difficult to ascertain whether it was the first or the second "bite" that contributed to the bleeding. This is a potential confounding factor in the analysis. Furthermore, bleeding can continue for $48 \mathrm{~h}$, and even longer, after biopsy, making it difficult to decide which technique has contributed to the bleeding. Therefore, it becomes important to use a protocol in which the hot and cold biopsies are performed in alternate patients rather than alternate biopsies in the same patient. If we follow this design, a semi-quantitative assessment can be made by quantifying the amount of saline instilled and the return amount and comparing between the two. This would also complement the qualitative assessment made by the bronchoscopist.

In addition, the authors have stated minimal damage even with cold biopsies. Whether this is an effect of the previous hot biopsy is also unclear, as multiple biopsies have been taken from the same lesion. Due to the small sample size, and given the fact that previous studies have shown significant pathological changes in the tissues after endobronchial electrocoagulation $[2,3]$, further investigation is required to confirm the findings of TREMBLAY et al. [1].

\section{A. Nath, R. Srinivas and R. Agarwal}

*Dept of Pulmonary Medicine, Postgraduate Institute of Medical Education and Research, Sector-12, Chandigarh, India.

\section{REFERENCES}

1 Tremblay A, Michaud G, Urbanski SJ. Hot biopsy forceps in the diagnosis of endobronchial lesions. Eur Respir J 2007; 29: 108-111.

2 Verkindre C, Brichet A, Maurage CA, Ramon P, Homasson JP, Marquette $\mathrm{CH}$. Morphological changes induced by extensive endobronchial electrocautery. Eur Respir J 1999; 14: 796-799.

3 van Boxem TJ, Westerga J, Venmans BJ, Postmus PE, Sudetja TG. Tissue effects of bronchoscopic electrocautery: bronchoscopic appearance and histologic changes of bronchial wall after electrocautery. Chest 2000; 117: 887-891.

DOI: 10.1183/09031936.00007707

\section{From the authors:}

We would like to thank A. Nath, R. Srinivas and R. Agarwal for their interest in our study [1], and their comments regarding our study design. Their main concerns centre around the study design, in which alternative biopsies of the same endobronchial lesion were taken with "hot" and "cold" biopsy forceps, with and without electrocoagulation, respectively.

The alternative study design would have been to randomise patients to receive either only hot or only cold biopsies. This study design was chosen for two primary purposes. First, the primary study outcome of pathological diagnosis is best compared when the same lesions are biopsied with the two techniques. Secondly, using the same patient/lesion as their own control allows the use of paired statistical analytical techniques, which are more powerful than unpaired analysis.

A. Nath and co-workers express concern regarding our finding that minimal coagulation damage was found with the hot biopsies. The quoted studies on electrocoagulation effect on airways are, in fact, what led us to carry out this study. Although A. Nath and co-workers comment on the "small sample size" of our study, it was powered to look for a decrease in biopsy yield from $95 \%$ to $70 \%$, as described in our methods, which may be an underestimate of power given the unpaired statistics that we used in this calculation. If a smaller difference in yield is felt to be clinically relevant, A. Nath and co-workers are correct that this study may not have detected such a difference. We stand by this conclusion as the samples were blindly reviewed by a pathologist who noted no such changes, while diagnostic rates were as high with the hot versus cold biopsies (slightly higher in fact). It is believed that with the use of monopolar forceps, the electrical current does not pass through the section of tissue inside the biopsy forceps, as the electrical resistance is lower if the current continues along the tip of the forceps and into the tissues, in essence protecting the sample from the current.

Quantification of bleeding is a more subjective and difficult outcome to measure. We had considered blinded assessment from video recording or simply measuring the amount of blood suctioned through the bronchoscope, but these approaches were not felt to remedy the problem. We agree that a randomisation approach in which biopsies were performed in different patients may have made this outcome measure more robust, but given that this was a secondary endpoint we stand by our study design as discussed previously. Practically speaking, although patients may have trace haemoptysis for 24-48 h after bronchoscopy, we believe this is due to old blood being expectorated rather than ongoing bleeding. During the study, we ensured that any bleeding had abated prior to proceeding to the next biopsy. In addition, given the alternative hot/cold design, any bias in bleeding assessment would occur in each group and the chance of misclassifying a clinically significant grade 3 or 4 bleed would appear very unlikely.

\section{A. Tremblay}

*Dept of Medicine, Divisions of Respiratory Medicine and Medical Oncology, Health Sciences Centre, Calgary, AB, Canada.

\section{STATEMENT OF INTEREST}

None declared.

\section{REFERENCES}

1 Tremblay A, Michaud G, Urbanski SJ. Hot biopsy forceps in the diagnosis of endobronchial lesions. Eur Respir J 2007; 29: 108-111.

DOI: $10.1183 / 09031936.00018307$ 